\title{
The Effect and Characteristic of Electromagnetically Resonance Mediated Clotrimazole on the Growth of Saccharomyces Cerevisiae
}

\author{
Jie Teng\#, Qing Li”, Miao Tian, Peng Gao, Hong Wu* and Bruce Qing Tang* \\ Institute of Life Science \& Technology,ENN Group, Langfang, Hebei, China \\ \#These authors contributed equally to this work \\ *Corresponding author: B Qing Tang, Institute of Life Science \& Technology, ENN Group, Hebei, China \\ Hong Wu, Institute of Life Science \& Technology, ENN Group, Hebei, China
}

\section{ARTICLE INFO}

Received: 幽 September 25, 2020

Published: October 02, 2020

Citation: Jie Teng, Qing Li, Miao Tian, Peng Gao, Hong Wu, Bruce Qing Tang. The Effect and Characteristic of Electromagnetically Resonance Mediated Clotrimazole on the Growth of Saccharomyces Cerevisiae. Biomed J Sci \& Tech Res 30(5)-2020. BJSTR. MS.ID.005023.

\section{ABSTRACT}

As a vibrational energy field, electromagnetic resonance (EMR) gives effect to all the substances composed of molecules, atoms and other particles with vibrational properties. In our previous study, we reported that clotrimazole (CLT) mediated by EMR inhibited the growth of Saccharomyces cerevisiae on solid media. This study confirmed that the inhibition effect also existed in liquid media. Based on the analysis of the experimental data of recent two years, the seasonal regular pattern of EMR mediated CLT was summarized.

Keywords: Electromagnetically Resonance; Clotrimazole; S. cerevisiae; Growth

Abbreviations: CLT: Clotrimazole; EMR: Electromagnetically Resonance; DDS: Direct Digital Synthesizer; OD: Optical Density

\section{Introduction}

Because molecules, atoms, electrons and chemical bonds all have vibrational properties, all organisms will be affected by the vibrational energy field [1]. EMR is a vibration energy field that affects the physiological state of organisms [2]. The information of matter can be remotely transmitted through EMR, affecting the physiological and biochemical characteristics of microorganisms [3-6]. Although this is a very important phenomenon, there are few related research papers yet. More research should be carried out to prove this phenomenon by more experimental evidence, and the mechanism needs to be analyzed. This will help us better understand the interaction between material information and organisms. CLT can inhibit the synthesis of ergosterol in fungal cell membrane and is widely used in the treatment of fungal infections [7]. We reported previously that CLT mediated by EMR inhibited the growth of $S$. cerevisiae on solid medium [2]. In this study, we investigated the effect in liquid medium, and then analyzed the seasonal regular pattern.

\section{Materials and Methods}

\section{Strain and Yeast Culture}

The yeast $S$. cerevisiae were obtained from ANGEL YEAST CO. LTD (China, 5g*400/F, Industrial instant dry yeasts). Yeast was cultured in rich medium YPD (1\% yeast extract, 2\% tryptone, 2\% $\mathrm{D}$-(+)-glucose) at $30{ }^{\circ} \mathrm{C}$ in shaker with $180 \mathrm{rpm}$ for $16 \mathrm{~h}$ used for experiment.

\section{Electromagnetically Resonance (EMR) Apparatus}

In this study, the same SET of EMR devices as in previous studies were used [2], the main features are as follow. The EMR apparatus consists of two standard pancake Tesla coils being made on printed circuit board that functioning as a transmitter and a receiver respectively. The secondary coil is printed on the top of the printed circuit board with $16 \mathrm{~m}$ total length, $0.5 \mathrm{~mm}$ width and $0.5 \mathrm{~mm}$ space between two wires. The center of the secondary coil 
is connected to a stainless-steel ball of $120 \mathrm{~mm}$ diameter. A ground wire is used to link the transmitter and receiver coil connecting the outside end of the secondary coil. The primary coil is printed under the printed circuit board with the same width and space, but shorter length [8] and connected directly to the DDS (Direct Digital Synthesizer) generator, whose output impedance is usually under $10 \mathrm{Ohms}$. When the two coils meet the condition of same frequency, same wave shape and opposite phase shift, self-resonance of the whole system will be generated. In our experiments, the resonant frequency varied between 3.2 and $3.6 \mathrm{MHz}$ due to the field attenuation by the flasks and the glass bottles containing the yeast or drug powder.

\section{Experimental Design}

Glass bottle with or without CLT powder was placed on the transmitter coil. For liquid culture, the logarithmic phase $S$. cerevisiae cells were diluted to about $1 \times 10^{2}$ cells $/ \mathrm{ml}$ in $50 \mathrm{ml}$ flask with liquid medium. Triplicate flasks were placed on the receiver coil for static culture. Another triplicate flask was placed outside the EMR field as Control. For CLT directly acting, the CLT concentration was $15 \mathrm{ug} / \mathrm{l}$. After $28 \mathrm{~h}$ of culture, optical density (OD) was measured at $600 \mathrm{~nm}$ (UV-2800, UNICO, shanghai, China) directly or by dilution with sterilized deionized water. The inhibition rate was calculated compared to the control, which was evaluated as: Inhibition rate $=$ (ODsample / ODcontrol-1) $\times 100 \%$.

\section{Results}

\section{Effect of EMR Mediated CLT on Yeast Growth in Liquid Culture}

Compared with the control group, the growth of yeast in EMR mediated CLT group was significantly inhibited. The inhibition rate was $16 \%$ at $28 \mathrm{~h}$, which was nearly half of the inhibition effect of adding CLT directly to yeast. In the empty bottle group, the yeast growth was significantly promoted to $36.5 \%$ increase at $28 \mathrm{~h}$ (Figure 1).

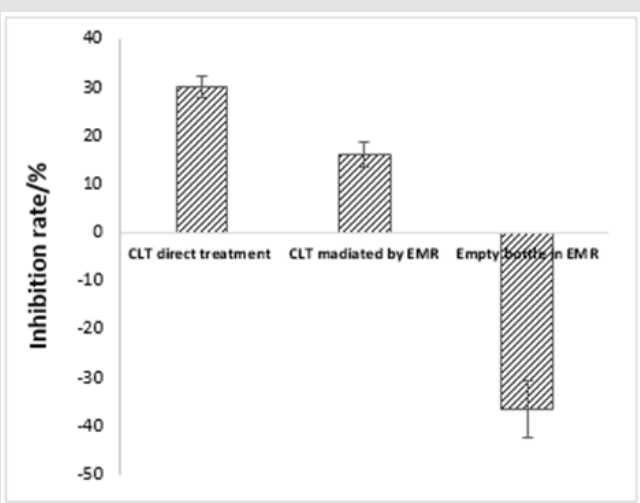

Figure 1: Effects of different treatments on yeast growth. Data were expressed by the average inhibition rate of the triplicate samples

\section{Seasonal Regular Pattern of EMR Mediated CLT on Yeast Growth}

Based on the summary of experimental data in recent two years, we analyzed the inhibitory effect of EMR mediated CLT on yeast growth. It was found that the inhibition effect gradually increased from spring to summer, reached the peak in August, and then began to decline (Figure 2).

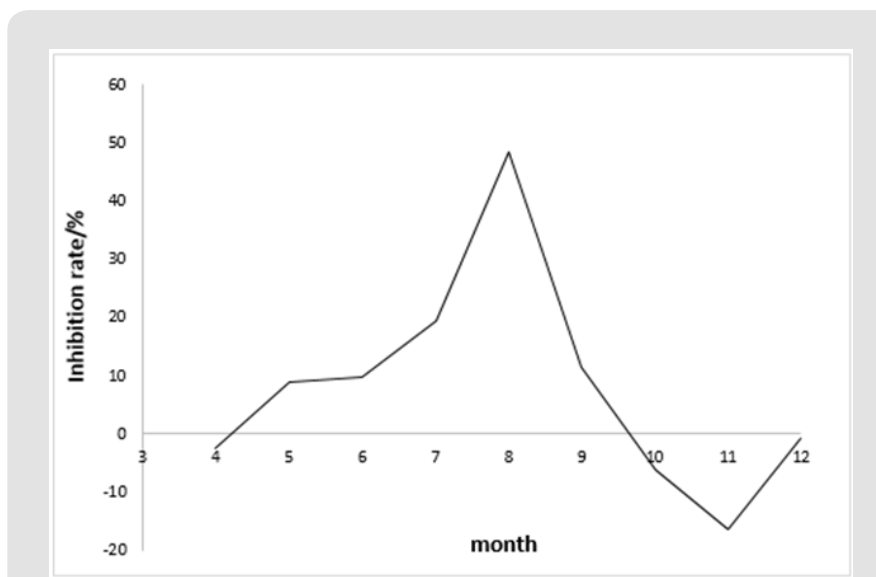

Figure 2: Seasonal regular pattern of EMR mediated CLT. Data is the average inhibition rate of each month

\section{Discussion}

EMR mediated CLT inhibited the growth of yeast cells in liquid medium, and the inhibition rate was 16\%. EMR (empty bottle) promoted the growth of yeast cells in liquid medium with the promotion rate of $36.5 \%$. These results indicate that EMR mediated CLT can play a role in both solid and liquid environments. All chemical substances have intrinsic electromagnetic characteristics, which contain the information and function of the substance. These characteristics can be separated from matter and transferred through physical field [9-12]. EMR plays the role of transmission. The molecular structure of CLT contains many ring structures, so it may act as a "ring antenna". This antenna structure is more conducive to the transmission of material information [6], and then EMR mediated CLT can inhibit the growth of yeast cells.

Water is considered to play a crucial role in information transmission and storage [8,11-13]. The dissipative nanostructures can be produced in the water system induced by the external physical field, and the drug information can be stored, translated and transferred through the structure of the water system, and its activity can be selectively regulated $[4,14,15]$. Whether in solid or liquid medium, water is the main component. EMR and CLT information mediated by EMR can affect the internal and external water environment of yeast cells, and then affect cell metabolism and proliferation $[3,5,16]$. We summarized the experimental results and found the seasonal regular pattern of EMR mediated CLT, this pattern is very consistent with Michal C's report [17]. This periodic 
change may be affected by the periodic electromagnetic field in the surrounding environment and may be related to the periodicity of the solar-earth system. This phenomenon needs further study.

EMR can change the water environment around the organism, and then remotely transmit the material information to act on the organism. A new approach could be developed to treat diseases, which would bring subversive innovation for modern medicine.

\section{References}

1. Oschman JL (2000) Energy Medicine: The Scientific Basis: Churchill Livingstone.

2. Qing L, Jie T, Miao T, Peng G, Li T, et al. (2019) Growth of Saccharomyces cerevisiae Influenced by Electromagnetically Resonance Mediated Clotrimazole.

3. Alberto F, Mario L, Sara P, Settimio G, Antonella L, et al. (2014) Electromagnetic information delivery as a new tool in translational medicine. Int J Clin Exp Med 7(9): 2550-2556.

4. Heredia-Rojas JA, Villarreal-Treviño L, Rodríguez-De Fuente AO, Herrera Menchaca LI, Gomez-Flores R, et al. (2015) Antimicrobial effect of vancomycin electrotransferred water against methicillin-resistant Staphylococcus aureus variant. Afr J Tradit Complement Altern Med 12(1): 104-108.

5. Rad I, Jalali K (2018) Electronic transmission of antibacterial property into water at extremely low frequency range: a preliminary study. J Altern Complement Med 24(5): 431-438.

6. Ebbers JA, Meyl K (2014) Drug effects in yeast mediated by scalar waves. Med Sci 8(30): 58-62.

7. Heredia-Rojas JA, Torres-Flores AC, Rodriguez-De la Fuente AO, MataCardenas BD, Rodriguez-Flores LE, et al. (2011) Entamoeba histolytica and Trichomonas vaginalis: Trophozoite growth inhibition by metronidazole electro-transferred water. Exp Parasitol 127(1): 80-83.

8. Montagnier L, Aissa J, Capolupo A, Craddock TJA, Kurian P, et al. (2017) Water bridging dynamics of polymerase chain reaction in the gauge theory paradigm of quantum fields. Water 9(6): 339.

9. Foletti A, Ledda M, D’Emilia E, Grimaldi S, Lisi A, et al. (2012) Experimental finding on the electromagnetic information transfer of specific molecular signals mediated through the aqueous system on two human cellular models. J Altern Complement Med 18(3): 258-261.

10. Kim WH (2013) New approach controlling cancer: water memory. J Vortex Sci Technol 1(1): 104.

11. Montagnier L, Aissa J, Del Giudice E, Lavallee C, Tedeschi A, et al. (2010) DNA waves and water. Quantitative Biol 306: 1-10.

12. Montagnier L, Del Giudice E, Aissa J, Lavallee C, Motschwiller S, et al. (2015) Transduction of DNA information through water and electromagnetic waves. Electromagnet Biol Med 34(2): 106-112.

13. Tang BQ, Li T, Bai X, Zhao M (2019) Rate limiting factors for DNA transduction induced by weak electromagnetic field. Electromagnet Biol Med 38(1): 55-65.

14. Jerman I, Ruzic R, Krasovec R, Metod Skarja (2005) Electrical transfer of molecule information into water, its storage, and bioeffects on plants and bacteria. Electromagn Biol Med 24(3): 341-353.

15. Widom A, Srivastava Y, Valenzi V (2010) The biophysical basis of benveniste experiments: entropy, structure, and information in water. Int J Quantum Chem 110(1): 252-256.

16. Ke YL, Chang FY, Chen MK, Li SL, Jang LS, et al. (2013) Influence of electromagnetic signal of antibiotics excited by low frequency pulsed electromagnetic fields on growth of escherichia coli. Cell Biochem Biophys 67(3): 1229-1237.

17. Michal C, Jeremy ZF, Ashkan F (2011) Electromagnetic cellular interactions. Progress in Biophysics and Molecular Biology 10593): 223246.

\section{ISSN: 2574-1241}

DOI: $10.26717 /$ BJSTR.2020.30.005023

Hong Wu, Bruce Qing Tang. Biomed J Sci \& Tech Res

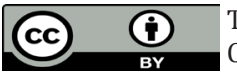

This work is licensed under Creative Commons Attribution 4.0 License

Submission Link: https://biomedres.us/submit-manuscript.php

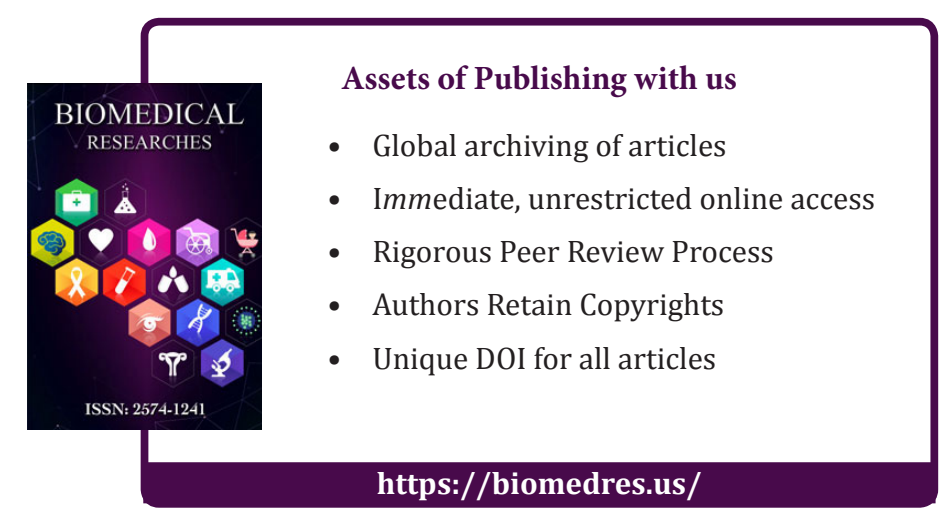

